\title{
Muon's Escalating Challenge to the Standard Model
}

\author{
Measurements of the muon magnetic moment strengthen a previously \\ reported tension with theoretical predictions, ushering in a new era of \\ precision tests of the standard model.
}

By Priscilla Cushman

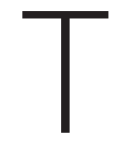

wenty years ago, the Brookhaven Muon $g-2$ experiment measured a value of the anomalous magnetic moment of the muon that disagreed by several parts per million with calculations based on the standard model (SM) of particle physics [1]. Physicists had long understood that the SM was incomplete, but the Muon $g-2$ experiment provided a measurable discrepancy between a very precise quantum-mechanical calculation and an equally precise measurement of a fundamental constant. The 2.7-sigma discrepancy was exciting in an era when the only news coming out of other major facilities-from the Large Electron-Positron Collider to the Tevatron-was how well the SM worked. Data

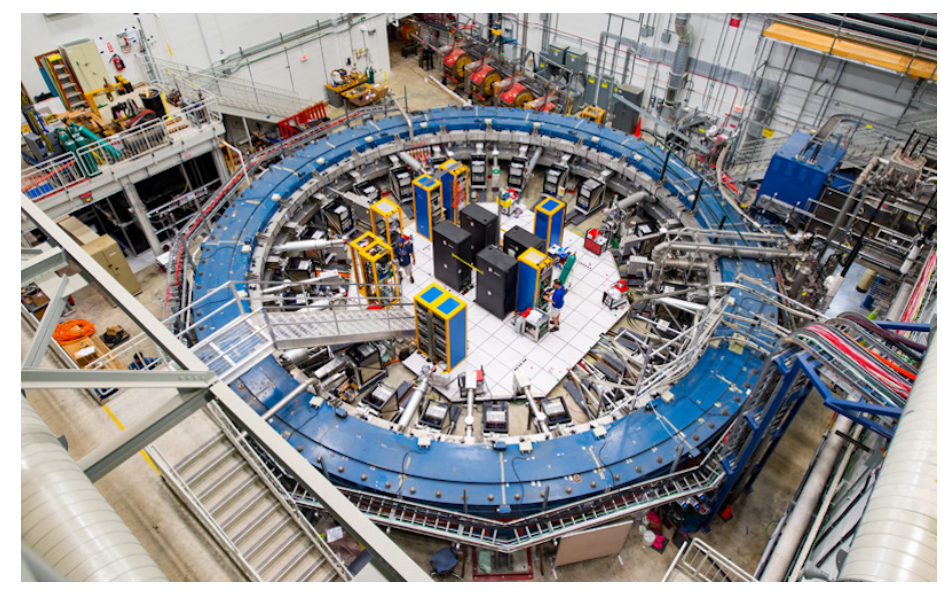

Figure 1: View of Fermilab's Muon $g-2$ ring, where the precession of muons in a magnetic field is used to measure the muon magnetic moment.

Credit: Reidar Hahn/Fermilab taking was halted after the 2001 run, since reducing significantly the statistical uncertainty would have required a higher-intensity muon beam, and reducing the systematic uncertainty would have required major engineering upgrades [2]. Meanwhile, theorists continued to improve the accuracy of the calculation, which increased the significance of the mismatch to 3.7 sigma as of 2020 [3]. It was clearly time to revisit the experimental side of the dilemma.

Today, the next-generation Muon $g-2$ Experiment at Fermi National Accelerator Laboratory (Fermilab) releases its first results [4], confirming the mean value of the discrepancy found two decades earlier. With this new independent measurement, the world average now stands at a more convincing 4.2-sigma departure from the SM. This mismatch could be the effect of new particles and new interactions that are considered by many natural SM extensions, such as supersymmetry, dark matter, and heavy neutrinos.

The magnetic moment of a particle is proportional to its spin and to its $g$-factor, which is exactly 2 for a point particle with half-integer spin. However, the muon is constantly interacting with virtual particles, which wink in and out of existence with quantum-mechanical probabilities calculable to an incredible precision. This fluctuating cloud of particles modifies the $g$-factor. The amount by which it differs from 2 is characterized by the anomaly $a=(g-2) / 2$, which is why the experiment is called Muon $g-2$. If the muon-a quantum-mechanical spinning top-is placed in a magnetic field, its spin will precess about the field direction at a frequency that depends on the charge distribution of those virtual particles. Measuring the 
precession frequency provides a determination of the anomaly and thereby of the overall effect of the virtual particles.

The Fermilab Muon $g-2$ Experiment (Fig. 1) follows the same technique used at Brookhaven. Polarized muons, whose spins are aligned with their direction of motion, are injected into a 14.2-m-diameter storage ring where they circle thousands of times thanks to their relativistically-stretched lifetime. As they decay, the muons spit out positrons that are detected by calorimeters lining the inner circumference of the ring. Inside the ring, the 1.45-T magnetic field that keeps the muons traveling in a circle also provides the magnetic torque that causes spin precession.

If $g$ were exactly 2 , the precession period would equal the cyclotron period, and the muon spin direction would rotate in lockstep with the muon momentum vector. Instead, the spin direction gradually gets out of sync, taking about 27 turns before realigning with the momentum. This frequency difference is the anomalous precession $\omega_{a}$. Since the decay positrons are preferentially emitted in the direction of the muon spin, their spectrum shifts to higher energies when the spin direction is aligned with the momentum. This shift produces a modulation in the number of positrons detected by the calorimeters.

The storage ring is a marvel of modern engineering. A concentric pair of superconducting coils creates a uniform vertical field inside the ring, while four electrostatic quadrupole plates serve as focusing elements. The design of the ring was optimized for muons with a momentum of $3.09 \mathrm{GeV} / c$, since at this "magic momentum" the electrostatic quadrupoles do not disturb the precession frequency to first order. This feature provided such an advantage that researchers opted to keep the original Brookhaven ring instead of exploring other design options. In 2013, the ring was dismantled, and the delicate superconducting coils were shipped over sea and land from Brookhaven to Fermilab [5]. Thanks to precision positioning of the 72 individual pole pieces and to the addition of programmable current shims, the reassembled magnet achieved a threefold improvement in azimuthal field uniformity over its earlier incarnation at Brookhaven.

The anomaly can be written as the ratio of two frequencies, measured to high precision by the experiment. The first is the measurement of the anomalous precession $\omega_{a}$ derived from analysis of the calorimeter response [6]. The second is a measurement of the magnetic field [7] along the muons' path, derived from the precession frequency $\omega_{p}$ of protons contained in fixed and moveable NMR probes (see Measuring the Field that Measures the Muon). Deriving both frequencies requires extremely precise knowledge of the muon beam dynamics [8], since each muon path samples a slightly different magnetic field and each calorimeter measures a positron modulation averaged over different muon orbits. The researchers obtained such information with extensive simulations, with corrections for eddy currents and mechanical vibrations, and with the use of data from straw chambers that tracked the positrons back to their muon parents. The anomaly can then be written in terms of the ratio $R^{\prime}=\omega_{a} / \tilde{\omega}_{p}^{\prime}$, where $\tilde{\omega}_{p}^{\prime}$ is a calibrated, muon-weighted, and magnetic-field-averaged frequency derived from $\omega_{p}$. The obtained precisions for the numerator and for the denominator of $R^{\prime}$ (438 ppb and $56 \mathrm{ppb}$, respectively) resulted in a $460-p p b$ precision in the determination of the anomaly.

The fact that $R^{\prime}$ is a ratio of frequencies led to a novel blinding process designed to prevent the researchers from subconsciously steering the analysis to favor a particular answer. Two gatekeepers from outside the collaboration applied a secret frequency offset to a clock used to calibrate $\omega_{a}$, revealing the value of the offset only after the data analysis was completed [9]. Confirmation of the previous result was never a foregone conclusion. As someone who has gone through a similar process at Brookhaven, I know how exciting-and somewhat terrifying-it can be to finally un-blind your data. Once you "open the box," you can neither retract nor revise your answer, so you have to trust that all the systematic sources of error have been accounted for.

Fermilab's new results provide compelling evidence that the answer obtained at Brookhaven was not an artifact of some unexamined systematics but a first glimpse of beyond-SM physics. While the results announced today are based on the 2018 run, data taken in 2019 and 2020 are already under analysis. We can look forward to a series of higher-precision results involving both positive and negative muons, whose comparison will provide new insights on other fundamental questions, from $C P T$ violation to Lorentz invariance [10]. This future muon $g-2$ campaign will lead to a fourfold 
improvement in the experimental accuracy, with the potential of achieving a 7-sigma significance of the SM deficit.

Other planned experiments will weigh in over the next decade, such as the E34 experiment at J-PARC, which employs a radically different technique for measuring $g-2$ [11]. E34 will also measure the muon electric dipole moment, offering a complementary window into SM deviations. In addition, the muon $g-2$ anomaly is not alone in suggesting "cracks" in the SM. These cracks have just gotten wider with LHCb's 3.1-sigma observation of the breakdown of lepton universality in beauty-quark decays [12]. Each glimpse of such anomalies (see The Era of Anomalies) provides new clues to the ultimate physics that governs our Universe, for which the SM is just the best "effective theory" up until now.

Priscilla Cushman: School of Physics and Astronomy, University of Minnesota, Minneapolis, MN, USA

\section{REFERENCES}

1. G. W. Bennett et al. (Muon g-2 Collaboration), "Final report of the E821 muon anomalous magnetic moment measurement at BNL," Phys. Rev. D 73, 072003 (2006).

2. J. Grange et al., "Muon (g-2) Technical Design Report," arXiv:1501.06858.

3. T. Aoyama et al., "The anomalous magnetic moment of the muon in the Standard Model," Phys. Rep. 887, 1 (2020).

4. B. Abi et al. (Muon g-2 Collaboration), "Measurement of the positive muon anomalous magnetic moment to $0.46 \mathrm{ppm}$," Phys. Rev. Lett. 126, 141801 (2021).

5. https://muon-g-2.fnal.gov/bigmove/.

6. T. Albahri et al. (Muon g-2 Collaboration), "Measurement of the anomalous precession frequency of the muon in the Fermilab Muon $g$ - 2 Experiment," Phys. Rev. D 103, 072002 (2021).

7. T. Albahri et al. (Muon g-2 Collaboration), "Magnetic-field measurement and analysis for the Muon $g-2$ Experiment at Fermilab," Phys. Rev. A 103, 042208 (2021).

8. T. Albahri et al. (Muon g-2 Collaboration), "Beam dynamics corrections to the Run-1 measurement of the muon anomalous magnetic moment at Fermilab," Phys. Rev. Accel. Beams 24, 044002 (2021).

9. https://www.youtube.com/watch?v=HtdVH1Wp7fs\&t=74s.

10. R. Bluhm et al., "CPT and Lorentz Tests with Muons," Phys. Rev. Lett. 84, 1098 (2000); R. Bluhm et al., "Testing CPT with Anomalous Magnetic Moments," Phys. Rev. Lett. 79, 1432 (1997).

11. M. Abe et al., "A new approach for measuring the muon anomalous magnetic moment and electric dipole moment," Prog. Theor. Exp. Phys. 2019 (2019).

12. R. Aaij et al. (LHCb Collaboration), "Test of lepton universality in beauty-quark decays," arXiv:2103.11769. 